\title{
Irrespeto y maltrato durante el parto y el aborto en América Latina: revisión sistemática y metaanálisis
}

\author{
Constanza Tobasía-Hege, ${ }^{1}$ Mariona Pinart, ${ }^{2}$ Sofia Madeira, ${ }^{3}$ Alessandra Guedes, ${ }^{1}$ Ludovic \\ Reveiz, ${ }^{1}$ Rosario Valdez-Santiago, ${ }^{4}$ Vicky Pileggi, ${ }^{3}$ Luz Arenas-Monreal, ${ }^{4}$ Anabel Rojas-Carmona, ${ }^{4}$ \\ Maricela Piña-Pozas, ${ }^{4}$ Rodolfo Gómez Ponce de León ${ }^{6}$ y João Paulo Souza ${ }^{3}$
}

Forma de citar

Tobasía-Hege C, Pinart M, Madeira S, Guedes A, Reveiz L, Valdez-Santiago R, et al. Irrespeto y maltrato durante el parto y el aborto en América Latina: revisión sistemática y metaanálisis. Rev Panam Salud Publica. 2019;43:e36. https://doi.org /10.26633/RPSP.2019.36

RESUMEN

Objetivo. Esta revisión sintetiza la evidencia cuantitativa, general y desglosada por categorías tipológicas de la falta de respeto y maltrato en la atención institucional del parto y el aborto en América Latina y el Caribe.

Métodos. Mediante búsquedas sistemáticas se identificaron 18 estudios primarios. Se calcularon $Q$ e $I^{2}$ y se realizaron metaanálisis, metarregresiones y análisis de subgrupos con la aplicación de un modelo de Der Simonian-Laird de efectos aleatorios agrupados con varianza inversa y la transformación arco-seno doble de Freeman-Tukey.

Resultados. Se identificaron estudios realizados en cinco países de América Latina. No se identificaron estudios del Caribe. La prevalencia agregada de falta de respeto y maltrato durante el parto y el aborto fue de $39 \%$. La medida agregada para este fenómeno durante el parto fue de $43 \%$ y la medida agregada en los casos de aborto fue de 29\%. La heterogeneidad elevada no permitió generar medidas agregadas según categorías tipológicas. No obstante, se presentan las frecuencias de formas específicas del fenómeno agrupadas tipológicamente.

Conclusiones. La evidencia sugiere que la falta de respeto y maltrato durante la atención del parto y el aborto son problemas de derechos humanos y salud pública prevalentes en algunos países de la Región. Es necesario lograr consenso internacional sobre la definición y operacionalización de este problema y desarrollar métodos estandarizados para su medición. Lo anterior es imprescindible para el alcance de las metas de la Agenda 2030 relacionadas con la reducción de la morbimortalidad maternoperinatal y la eliminación de todas las formas de violencia y discriminación contra la mujer.

Palabras clave Violencia contra la mujer; parto humanizado; servicios de salud para mujeres, aborto; parto.

La Organización de las Naciones Unidas ha enfatizado que la morbimortalidad materna es un problema grave de salud pública y un signo de discriminación que menoscaba el desarrollo de las naciones, y ha hecho un llamamiento a garantizar el derecho a servicios de alta calidad para la atención del embarazo y el parto y a una atención de salud digna y respetuosa para todas las mujeres $(1,2)$. Esto ha situado la supervivencia materno-neonatal entre los desafíos sanitarios más críticos a nivel mundial y en el núcleo de las estrategias para el logro de varias metas de los Objetivos de Desarrollo Sostenible, en especial las

\footnotetext{
Organización Panamericana de la Salud/Organización Mundial de la Salud (OPS/OMS), Washington D.C., Estados Unidos de América. $\square$ Constanza Tobasía-Hege, hegecon@paho.org

2 Investigadora especialista en revisiones sistemáticas, Berlín, Alemania.

3 Departamento de Medicina Social de la Facultad de Medicina de Ribeirão Preto, Universidad de São Paulo (FMRP/USP), São Paulo, Brasil.
} 
relacionadas con la salud materna (metas 3.1 y 3.2), y la eliminación de todas las formas de violencia y discriminación contra las mujeres (meta 5.2) $(3,4)$. Sin embargo, varios estudios revelan que un gran número de embarazadas en todo el mundo sufren irrespeto y maltrato en la atención institucional del parto y del aborto, períodos críticos en el curso de la vida y la salud de las mujeres (5-10).

La Organización Mundial de la Salud (OMS) emitió una declaración en la que instaba con firmeza a los sistemas de salud a erradicar dicho maltrato, que representa una violación de los derechos humanos $(6,7,11)$ y del derecho de las mujeres al disfrute del nivel más alto posible de salud, perjudica la credibilidad y la confianza en los sistemas de salud, desalienta la utilización de los servicios ginecoobstétricos, disminuye la adherencia a los tratamientos y la adopción de medidas preventivas y menoscaba el bienestar integral de las mujeres y sus hijos $(6,10,11-15)$, en especial en los grupos poblacionales en situación de vulnerabilidad (16).

Aunque el fenómeno ha sido reconocido desde hace varios años como un problema que afecta la atención institucional del parto y el aborto, no existe aún consenso internacional sobre su definición y operacionalización $(15,17-26)$ y se desconoce su magnitud a nivel mundial y regional.

A pesar de las diferentes denominaciones del fenómeno, esta revisión emplea "irrespeto y maltrato", en consonancia con la terminología usada por la OMS (6) y contempla el parto vaginal y por cesárea y el aborto espontáneo e inducido. El objetivo es contribuir al conocimiento de su prevalencia en América Latina y el Caribe (ALC). Esto es indispensable para determinar su impacto en la salud maternoperinatal e identificar las intervenciones más efectivas para su abordaje.

\section{MATERIALES Y MÉTODOS}

El protocolo de esta revisión sistemática está registrado y publicado en PROSPERO (CRD42016038651) (27).

Se realizó una consulta sistemática de la literatura en EMBASE, Pubmed, LILACS y Scielo de acuerdo con la estrategia de búsqueda preestablecida. Además, se revisaron en forma manual las publicaciones Reproductive Health Matters, The Lancet, International Perspectives on Sexual and Reproductive Health y Revista Panamericana de Salud Pública, por destacarse en el tema, la Región o ambos; se buscó información relevante en documentos de la Organización Panamericana de la Salud (OPS) y la OMS; se revisaron las bibliografías de los estudios incluidos y se contactaron profesionales e instituciones relevantes.

\section{Búsquedas}

Se buscó la literatura publicada entre el 1 de enero de 1990 y el 4 de octubre de 2017. Se utilizó vocabulario controlado y con la combinación de al menos dos componentes principales: maternal health, perinatal health, childbirth, delivery o abortion, obstetric y mistreatment of women, violence, disrespect o abuse. No hubo restricción del idioma. La bibliografía se procesó utilizando EndNote, $X 7^{\circledR}$.

\section{Selección de los estudios}

Los títulos y resúmenes de los estudios, y los textos completos seleccionados fueron examinados por revisores independientes
(MP, SM, VP, CH) mediante la aplicación de los siguientes criterios de inclusión: (i) estudios primarios o secundarios con datos cuantitativos de prevalencia de irrespeto y maltrato en la atención institucional del parto y/o aborto, (ii) revisiones sistemáticas con datos cuantitativos acerca del tema de interés, (iii) estudios producidos desde 1990 hasta las fechas de consulta de cada una de las bases de datos, (iv) estudios cuyo texto completo fuese accesible, (v) estudios publicados en inglés, español o portugués, (vi) estudios realizados en países de ALC. Se excluyeron estudios que abordaron el fenómeno fuera del ámbito institucional (por ejemplo, parto domiciliario). Las discrepancias fueron discutidas entre los revisores o examinadas por un miembro diferente del equipo hasta lograr consenso (JPS).

\section{Evaluación de la calidad}

Se aplicó la herramienta de Hoy et al. (28) para la evaluación de riesgo de sesgo en estudios de prevalencia. Esta herramienta evalúa validez externa (criterios 1-4) e interna (criterios 5-10) bajo los siguientes dominios: sesgo de selección (criterios 1-3), sesgo de no respuesta (criterio 4), sesgo de medición (criterios 5-9) y sesgo relacionado con el análisis (criterio 10). El criterio 11 permitió calificar el riesgo como "bajo" con ocho o más criterios cumplidos, "moderado" con cinco a siete criterios y "alto" con cuatro criterios o menos. Dos miembros diferentes del equipo (MP y SM) realizaron la evaluación.

\section{Extracción de datos}

Se utilizaron formularios estandarizados que incluían los siguientes dominios: título, autores, país, año de publicación, referencia bibliográfica, idioma original, área de cobertura, diseño, metodología, métodos de recopilación de datos, características de las participantes, tipo de parto, tipo de institución de salud, tipo de personal de salud que atendió el parto o el aborto, tipo de personal de salud asociado a los eventos, duración total de la estancia hospitalaria, tipo y frecuencia del irrespeto y maltrato (MP, SM, VP).

\section{Síntesis de los datos}

Se extrajeron las prevalencias generales de cualquier forma de irrespeto y maltrato y se realizó combinación cuantitativa de las mismas usando la transformación arcoseno doble de Freeman-Tukey $(29,30)$. Se evaluaron las diferencias de prevalencia según el tipo de hospital, método de recolección de datos y tipo de atención obstétrica mediante el cálculo de la proporción del fenómeno para cada estudio junto con los intervalos de confianza asociados del 95\% (IC95\%) y con la utilización de un modelo de DerSimonian-Laird de efectos aleatorios agrupados con el método de varianza inversa. Los análisis estadísticos se realizaron con $\mathrm{R}$.

Además, se extrajeron las prevalencias específicas del fenómeno usando como referencia la clasificación tipológica de maltrato durante el parto descrita por Bohren et al. (8) que consta de siete categorías tipológicas (términos de tercer orden), desglosadas a su vez en subcategorías analíticas (términos de segundo orden) y descriptivas (términos de primer orden). Estas prevalencias desglosadas por tipo no fueron metaanalizadas. 


\section{Evaluación de la heterogeneidad}

La proporción de varianza debida a la heterogeneidad estadística se calculó usando Q e $\mathrm{I}^{2}$. $\mathrm{I}^{2}$ menor de $25 \%$ representó heterogeneidad baja; entre $25 \%$ y $50 \%$, moderada; entre $51 \%$ y $75 \%$, sustancial, y entre $76 \%$ y $100 \%$, elevada. La evaluación de las posibles fuentes de heterogeneidad se realizó mediante análisis de subgrupo y metarregresiones de efectos aleatorios univariadas por país: Brasil versus otros países, tipo de parto, tipo de hospital, método de recolección de datos y tipo de desenlace medido (trato deshumanizante e insatisfacción con la atención recibida vs. ocurrencia de alguna forma de irrespeto y maltrato). No se encontró una variable estadísticamente significativa para realizar metarregresiones multivariadas.

El informe de resultados siguió las pautas del PRISMA (Preferred Reporting Items for Systematic Review and Meta-Analysis) (31).

\section{RESULTADOS}

Las búsquedas en las bases de datos y en otras fuentes arrojaron 26118 estudios. Tras eliminar duplicados y analizar títulos y resúmenes, se seleccionaron 128 estudios para lectura completa. Se incluyeron un total de 18 estudios primarios $(15,20,21$, $23,25,32-44)$, de los cuales 12 se incluyeron en el metaanálisis (figura 1).

Los estudios se realizaron en cinco países: 12 estudios en Brasil $(15,20,23,25,32,33,36,38-42)$, un estudio en Chile (37), dos estudios en México $(34,35)$, un estudio en Perú (43) y dos estudios en Venezuela $(21,44)$. No se encontraron estudios desarrollados en el Caribe. Los trabajos fueron publicados entre 2005 y 2017 y, con excepción de uno de casos y controles (42), todos los estudios fueron de corte transversal. El tamaño de la muestra varió entre 78 (20) y 15688 mujeres (36), con un total de 37 028. Catorce investigaciones exploraron el fenómeno de interés en el contexto del parto vaginal y por cesárea. Dos estudios exploraron solo el aborto inducido $(20,41)$ y dos más abarcaron el parto vaginal y por cesárea y el aborto inducido y espontáneo $(33,44)$. Los métodos para la recolección de datos fueron entrevistas y revisiones del historial clínico de las mujeres (cuadro 1).

El riesgo de sesgo fue clasificado como bajo para tres estudios $(17 \%)$, moderado para nueve $(50 \%)$ y alto para seis $(33 \%)$. Ningún estudio satisfizo los diez criterios evaluados.

FIGURA 1. Diagrama de flujo del proceso de búsqueda e inclusión de estudios

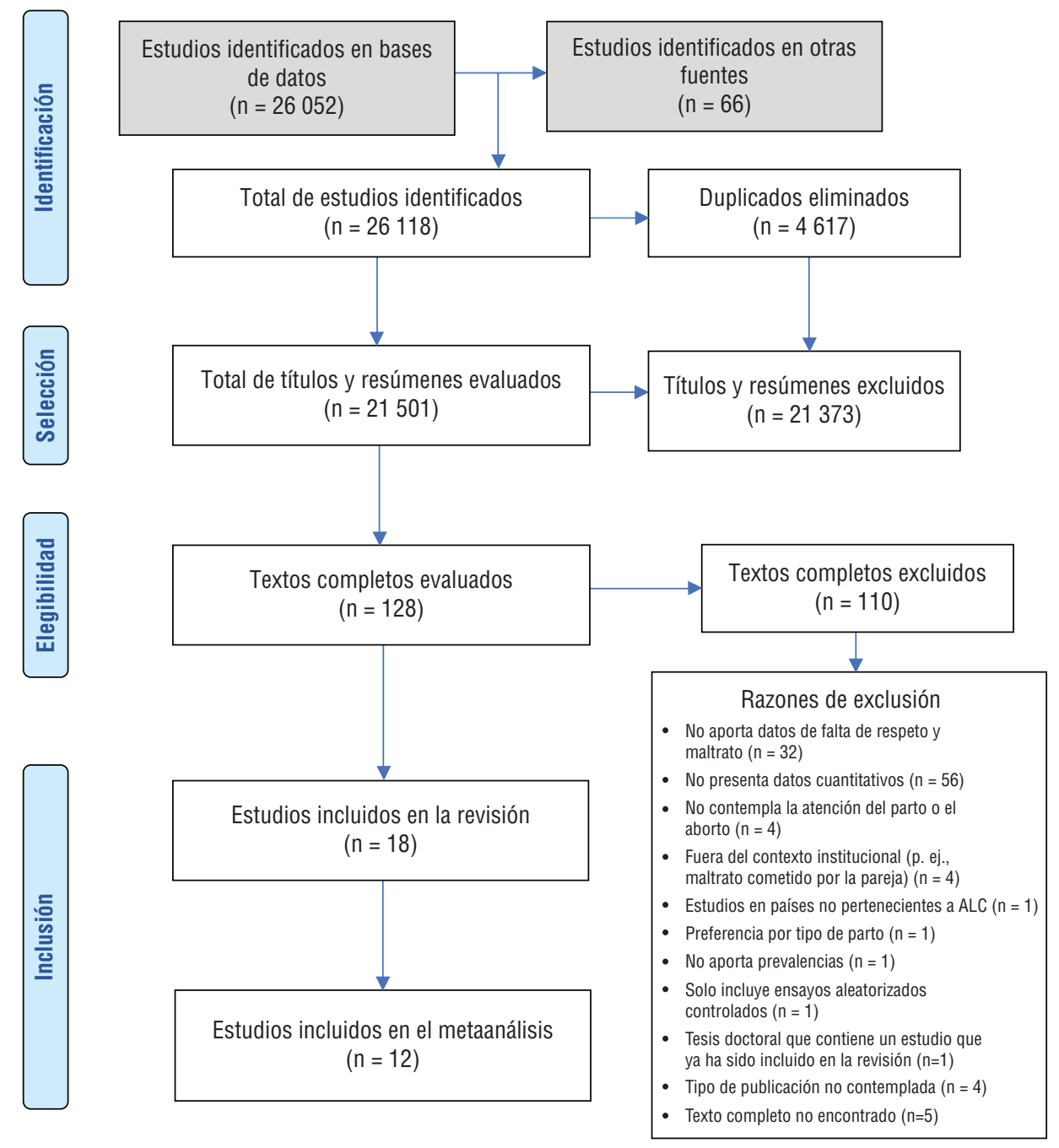

ALC, América Latina y el Caribe. 
CUADRO 1. Características de los estudios incluidos

\begin{tabular}{|c|c|c|c|c|c|c|c|c|c|}
\hline Estudio & País & $\begin{array}{l}\text { Diseño del } \\
\text { estudio }\end{array}$ & $\begin{array}{l}\text { Duración de } \\
\text { la recolección } \\
\text { de datos }\end{array}$ & Método de muestreo & $\begin{array}{l}\text { Tamaño de la } \\
\text { muestra (N) }\end{array}$ & $\begin{array}{l}\text { Instrumentos de } \\
\text { medida }\end{array}$ & $\begin{array}{l}\text { Tipo de parto } \\
0 \text { aborto }\end{array}$ & $\begin{array}{l}\mathrm{IM} / \mathrm{I} \\
(\mathrm{n})\end{array}$ & $\begin{array}{l}\text { Prevalencia } \\
(\%)\end{array}$ \\
\hline $\begin{array}{l}\text { Andrade } \\
2016(23)\end{array}$ & Brasil & Transversal & $\begin{array}{l}\text { Agosto a } \\
\text { diciembre de } \\
2014\end{array}$ & $\begin{array}{l}\text { Todas las mujeres que tuvieron } \\
\text { parto vaginal durante el de } \\
\text { estudio en un hospital de } \\
\text { referencia de Recife }\end{array}$ & 603 & $\begin{array}{l}\text { Entrevistas e } \\
\text { historiales médicos } \\
\text { de la mujer y del } \\
\text { recién nacido }\end{array}$ & $\begin{array}{l}\text { Vaginal } \\
(n=591) \\
\text { vaginal } \\
\text { instrumental } \\
(n=12)\end{array}$ & 522 & 86,6 \\
\hline $\begin{array}{l}\text { Aquino } \\
2012(41)\end{array}$ & Brasil & Transversal & $\begin{array}{l}\text { Agosto a } \\
\text { diciembre de } \\
2010\end{array}$ & $\begin{array}{l}\text { Censo de mujeres internadas } \\
\text { en siete hospitales de Salvador, } \\
\text { ocho de Recife y cuatro de } \\
\text { San Luis. Se incluyeron todos } \\
\text { los hospitales públicos con } \\
\text { internaciones por aborto }\end{array}$ & 2804 & $\begin{array}{l}\text { Entrevistas cara } \\
\text { a cara usando } \\
\text { cuestionarios } \\
\text { validados }\end{array}$ & $\begin{array}{l}\text { Aborto } \\
\text { inducido }\end{array}$ & 235 & 8,4 \\
\hline $\begin{array}{l}\text { Binfa } 2016 \\
\text { (37) }\end{array}$ & Chile & Transversal & $\begin{array}{l}\text { Setiembre de } \\
2010 \text { a junio } \\
\text { de } 2011\end{array}$ & $\begin{array}{l}\text { Se seleccionaron mujeres } \\
\text { (método no descrito) en } \\
\text { dos hospitales principales } \\
\text { pertenecientes al sistema } \\
\text { nacional de salud del área } \\
\text { metropolitana de Santiago }\end{array}$ & 508 & $\begin{array}{l}\text { Entrevistas cara a } \\
\text { cara mediante el uso } \\
\text { de cuestionarios } \\
\text { validados }\end{array}$ & $\begin{array}{l}\text { Parto vaginal } \\
(n=401) \text { y } \\
\text { cesárea } \\
(n=107)\end{array}$ & $141^{\mathrm{a}}$ & 27,8 \\
\hline $\begin{array}{l}\text { D'Orsi } 2005 \\
\text { (42) }\end{array}$ & Brasil & Caso-control & $\begin{array}{l}\text { Octubre de } \\
1998 \text { a marzo } \\
\text { de } 1999\end{array}$ & $\begin{array}{l}\text { Conveniencia. Se seleccionaron } \\
\text { el } 50 \% \text { de partos vaginales y } \\
\text { todos los partos por cesárea } \\
\text { ocurridos el día anterior a la } \\
\text { entrevista } \\
\text { Dos maternidades, una pública y } \\
\text { otra privada dentro del sistema } \\
\text { nacional de salud }\end{array}$ & 909 & $\begin{array}{l}\text { Entrevistas e } \\
\text { historiales médicos } \\
\text { de la mujer }\end{array}$ & $\begin{array}{l}\text { Parto vaginal } \\
(n=454) \text { y } \\
\text { cesárea } \\
(n=455)\end{array}$ & NRD & NRD \\
\hline $\begin{array}{l}\text { D'Orsi } 2014 \\
\text { (36) }\end{array}$ & Brasil & Transversal & $\begin{array}{l}\text { Marzo de } \\
2011 \text { a } \\
\text { febrero de } \\
2013\end{array}$ & $\begin{array}{l}\text { Se seleccionaron hospitales, } \\
\text { tanto públicos como privados, } \\
\text { con más de } 500 \text { partos/año }\end{array}$ & 15688 & $\begin{array}{l}\text { Entrevistas telefónicas } \\
\text { y cara a cara con } \\
\text { cuestionarios } \\
\text { validados e historiales } \\
\text { médicos de las } \\
\text { mujeres }\end{array}$ & $\begin{array}{l}\text { Parto vaginal } \\
(44,6 \%) \\
\text { y cesárea } \\
(53,4 \%)\end{array}$ & 922 & 5,9 \\
\hline $\begin{array}{l}\text { Montesinos } \\
\text { Segura } \\
2017(43)\end{array}$ & Perú & Transversal & $\begin{array}{l}\text { Abril a julio } \\
2016\end{array}$ & $\begin{array}{l}\text { El objetivo original fue el de } \\
\text { reclutar todos los hospitales de } \\
\text { todas las ciudades con un gran } \\
\text { número de habitantes } \\
\text { Sólo se obtuvieron permisos } \\
\text { de } 14 \text { hospitales públicos } \\
\text { localizados en nueve ciudades } \\
\text { del Perú }\end{array}$ & 1528 & $\begin{array}{l}\text { Cuestionario de } 36 \\
\text { ítems (Bowser y Hill } \\
\text { y otros estudios), } \\
\text { aplicado a a mujeres } \\
\text { en el posparto } \\
\text { inmediato (se realizó } \\
\text { una prueba piloto } \\
\text { para evaluar los } \\
\text { instrumentos) }\end{array}$ & $\begin{array}{l}\text { Parto vaginal } \\
(n=968) \text { y } \\
\text { cesárea } \\
(n=560)\end{array}$ & 1488 & 97,4 \\
\hline $\begin{array}{r}\text { Nagahama } \\
2008(39)\end{array}$ & Brasil & Transversal & $\begin{array}{l}\text { Marzo de } \\
2005 \text { a } \\
\text { febrero de } \\
2006\end{array}$ & $\begin{array}{l}\text { Censo de partos vivos en dos } \\
\text { hospitales del Sistema Único de } \\
\text { Salud (SUS) durante el período } \\
\text { de estudio }\end{array}$ & 569 & $\begin{array}{l}\text { Entrevistas con } \\
\text { preguntas abiertas } \\
\text { y cerradas (de } \\
\text { cuestionarios } \\
\text { validados) }\end{array}$ & $\begin{array}{l}\text { Parto vaginal } \\
(n=273) \text { y } \\
\text { cesárea } \\
(n=296)\end{array}$ & NRD & NRD \\
\hline $\begin{array}{l}\text { Narchi } 2010 \\
\text { (32) }\end{array}$ & Brasil & Transversal & $\begin{array}{l}\text { Enero a julio } \\
\text { de } 2008\end{array}$ & $\begin{array}{l}\text { Conveniencia. Entre usuarias } \\
\text { atendidas en dos instituciones } \\
\text { públicas de salud de la zona } \\
\text { oriental de la ciudad de San } \\
\text { Pablo }\end{array}$ & 90 & $\begin{array}{l}\text { Entrevistas con } \\
\text { preguntas abiertas } \\
\text { y cerradas (basadas } \\
\text { en instrumentos } \\
\text { validados) además de } \\
\text { revisión de historiales } \\
\text { médicos }\end{array}$ & $\begin{array}{l}\text { Parto vaginal } \\
(n=82) \text { y } \\
\text { cesárea } \\
(n=8)\end{array}$ & $76^{\mathrm{a}}$ & 84,4 \\
\hline
\end{tabular}




\section{CUADRO 1. (Cont.)}

\begin{tabular}{|c|c|c|c|c|c|c|c|c|c|}
\hline Estudio & País & $\begin{array}{l}\text { Diseño del } \\
\text { estudio }\end{array}$ & $\begin{array}{l}\text { Duración de } \\
\text { la recolección } \\
\text { de datos }\end{array}$ & Método de muestreo & $\begin{array}{l}\text { Tamaño de la } \\
\text { muestra (N) }\end{array}$ & $\begin{array}{l}\text { Instrumentos de } \\
\text { medida }\end{array}$ & $\begin{array}{l}\text { Tipo de parto } \\
\text { o aborto }\end{array}$ & $\begin{array}{l}\mathrm{IM} / \mathrm{I} \\
(\mathrm{n})\end{array}$ & $\begin{array}{l}\text { Prevalencia } \\
(\%)\end{array}$ \\
\hline $\begin{array}{l}\text { Nieto- } \\
\text { González } \\
2011 \text { (34) }\end{array}$ & México & Transversal & $\begin{array}{l}\text { En } 2010 \\
\text { (durante ocho } \\
\text { semanas) }\end{array}$ & $\begin{array}{l}\text { Muestra representativa (método } \\
\text { no descrito) de mujeres } \\
\text { embarazadas de segunda } \\
\text { gesta de más de } 22 \text { semanas } \\
\text { de embarazo obtenidas del } \\
\text { promedio mensual de mujeres } \\
\text { que acuden a la consulta prenatal } \\
\text { de un hospital general público de } \\
\text { Ciudad de México }\end{array}$ & 380 & $\begin{array}{l}\text { Encuestas } \\
\text { autoaplicadas } \\
\text { a mujeres que } \\
\text { voluntariamente } \\
\text { accedieron a } \\
\text { participar en el } \\
\text { estudio basadas } \\
\text { en instrumentos } \\
\text { validados }\end{array}$ & $\begin{array}{l}\text { Tipo de parto } \\
\text { reportado }\end{array}$ & 23 & 6,1 \\
\hline $\begin{array}{l}\text { Pereira } \\
2015 \text { (44) }\end{array}$ & Venezuela & Transversal & $\begin{array}{l}\text { Junio a } \\
\text { octubre de } \\
2012\end{array}$ & $\begin{array}{l}\text { Muestreo de carácter intencional } \\
\text { no probabilístico. Se incluyeron } \\
\text { mujeres hospitalizadas en el } \\
\text { departamento de obstetricia y } \\
\text { ginecología del Hospital General } \\
\text { del Oeste "Dr. José Gregorio } \\
\text { Hernández" (público) de Caracas, } \\
\text { Venezuela, para ser atendidas por } \\
\text { parto, cesárea o por presentar } \\
\text { aborto espontáneo }\end{array}$ & 326 & $\begin{array}{l}\text { Entrevista con un } \\
\text { cuestionario (no } \\
\text { validado) de } 15 \\
\text { preguntas cerradas } \\
\text { para saber si fueron } \\
\text { sometidas a cualquier } \\
\text { tipo de agresión física } \\
\text { o psicológica }\end{array}$ & $\begin{array}{l}\text { Partos } \\
\text { ( } \mathrm{n}=225, \\
\text { vaginal y } \\
\text { cesárea) } \\
\text { y aborto } \\
\text { espontáneo } \\
\text { ( } \mathrm{n}=101)\end{array}$ & $86^{b}$ & $26,4^{c}$ \\
\hline $\begin{array}{r}\text { Rodrigues } \\
2017 \text { (25) }\end{array}$ & Brasil & Transversal & $\begin{array}{l}\text { Noviembre } \\
2013 \text { a enero } \\
2014\end{array}$ & $\begin{array}{l}\text { La Red Cigüeña fue implantada } \\
\text { por el Ministerio de Salud en } \\
2011 \text { en hospitales públicos } \\
\text { pertenecientes al sistema único } \\
\text { de salud. En Fortaleza-Cascavel, } \\
\text { la Red Cigueña está compuesta } \\
\text { por } 11 \text { hospitales-maternidades } \\
\text { de media y alta complejidad }\end{array}$ & $293^{d}$ & $\begin{array}{l}\text { Cuestionario aplicado } \\
\text { a mujeres en el } \\
\text { posparto además de } \\
\text { revisión de historiales } \\
\text { médicos }\end{array}$ & Parto vaginal & NRD & NRD \\
\hline $\begin{array}{l}\text { Santos } \\
2008(40)\end{array}$ & Brasil & Transversal & $\begin{array}{l}\text { Julio a } \\
\text { diciembre de } \\
2006\end{array}$ & $\begin{array}{l}\text { Todas las mujeres que parieron } \\
\text { en un hospital público del interior } \\
\text { de San Pablo durante el período } \\
\text { de estudio }\end{array}$ & 279 & Historiales médicos & Parto vaginal & NRD & NRD \\
\hline $\begin{array}{l}\text { Souza } 2017 \\
(15)\end{array}$ & Brasil & Transversal & Durante 2011 & $\begin{array}{l}\text { Muestreo por conglomerados } \\
\text { en etapas múltiples } \\
\text { Se seleccionaron } 25 \text { centros de } \\
\text { salud en } 21 \text { de las } 30 \text { regiones } \\
\text { administrativas del distrito } \\
\text { federal }\end{array}$ & 10468 & $\begin{array}{l}\text { Entrevista con } \\
\text { cuestionario validado }\end{array}$ & $\begin{array}{l}\text { Parto vaginal } \\
(n=2774) \text { y } \\
\text { cesárea } \\
(n=7558)\end{array}$ & a & 2,6 \\
\hline $\begin{array}{l}\text { Terán } 2013 \\
\text { (21) }\end{array}$ & Venezuela & Transversal & $\begin{array}{l}\text { Mayo a } \\
\text { agosto de } \\
2011\end{array}$ & $\begin{array}{l}\text { Selección (no reporta el método } \\
\text { de selección) de usuarias } \\
\text { ingresadas en los servicios } \\
\text { de puerperio de la Maternidad } \\
\text { "Concepción Palacios" }\end{array}$ & 425 & $\begin{array}{l}\text { Encuestas } \\
\text { autoaplicadas } \\
\text { contestadas de forma } \\
\text { anónima }\end{array}$ & $\begin{array}{l}\text { Puerperio de } \\
\text { parto simple } \\
(n=249), \\
\text { parto } \\
\text { instrumental } \\
(n=9) \\
\text { cesárea } \\
(n=167)\end{array}$ & 210 & 49,4 \\
\hline $\begin{array}{l}\text { Valdez- } \\
\text { Santiago } \\
2013(35)\end{array}$ & México & Transversal & $\begin{array}{l}\text { Mayo a junio } \\
\text { de } 2012\end{array}$ & $\begin{array}{l}\text { Método no reportado } \\
\text { Se seleccionaron usuarias de } \\
\text { tres hospitales públicos del } \\
\text { estado de Morelos: Hospital } \\
\text { General de Tetecala, Hospital de } \\
\text { la Mujer de Yautepec y Hospital } \\
\text { General José G. Parres de } \\
\text { Cuernavaca }\end{array}$ & 512 & $\begin{array}{l}\text { Cuestionario aplicado } \\
\text { a mujeres en el } \\
\text { posparto inmediato } \\
\text { (se realizó una prueba } \\
\text { piloto para evaluar los } \\
\text { instrumentos) }\end{array}$ & $\begin{array}{l}\text { Parto vaginal } \\
(42,6 \%), \\
\text { cesárea } \\
(50 \%) \text { y } \\
\text { cesárea } \\
\text { programada } \\
(7,4 \%)\end{array}$ & 149 & 29,1 \\
\hline \multirow[t]{2}{*}{$\begin{array}{l}\text { Venturi } \\
2013(33)\end{array}$} & \multirow[t]{2}{*}{ Brasil } & \multirow[t]{2}{*}{ Transversal } & \multirow[t]{2}{*}{$\begin{array}{l}\text { Durante } \\
\text { agosto } 2010\end{array}$} & \multirow{2}{*}{$\begin{array}{l}\text { Mujeres atendidas en hospitales } \\
\text { públicos y privados de } 25 \\
\text { estados de Brasil durante el } \\
\text { período de estudio }\end{array}$} & 1411 & \multirow[t]{2}{*}{$\begin{array}{l}\text { Encuestas basadas } \\
\text { en instrumentos } \\
\text { validados }\end{array}$} & $\begin{array}{l}\text { Parto } \\
\text { (tipo no } \\
\text { reportado) }\end{array}$ & 353 & 25,0 \\
\hline & & & & & 28 & & $\begin{array}{l}\text { Aborto } \\
\text { inducido }\end{array}$ & 15 & 53,6 \\
\hline
\end{tabular}

IM/I (n), falta de respeto y maltrato/insatisfacción (número de eventos); NRD, no se reportaron datos.

a Descontento o insatisfacción con el trato recibido.

De los cuales: parto $(n=36)$, cesárea $(n=19)$ y aborto $(n=31)$.

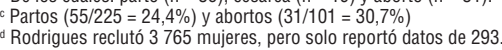




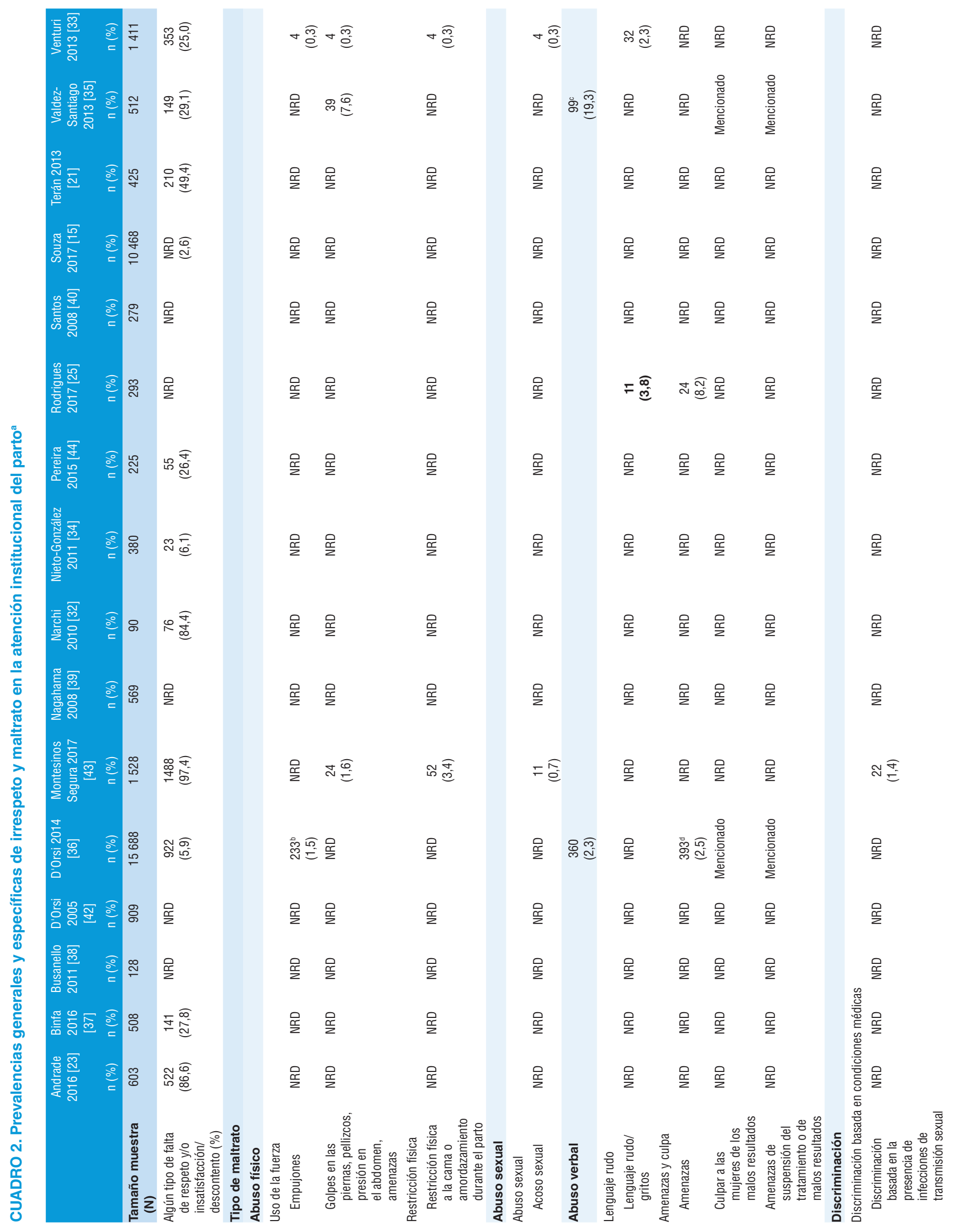




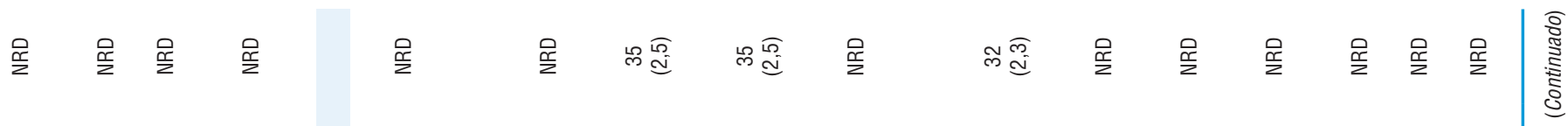

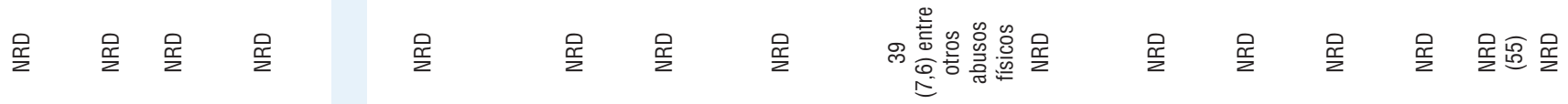

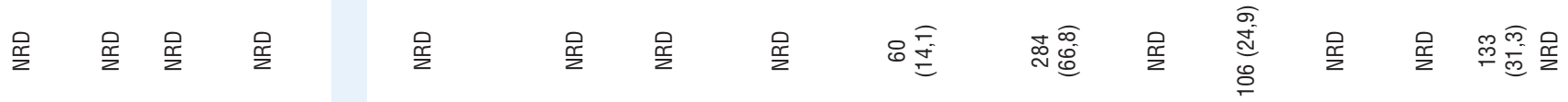

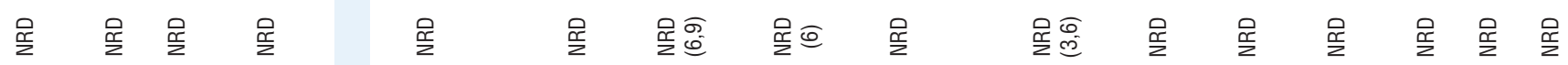

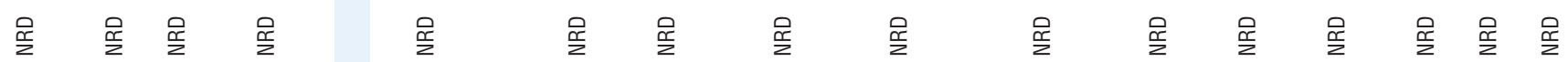

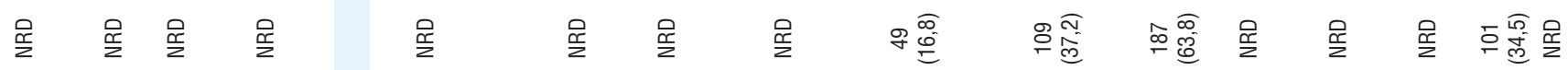

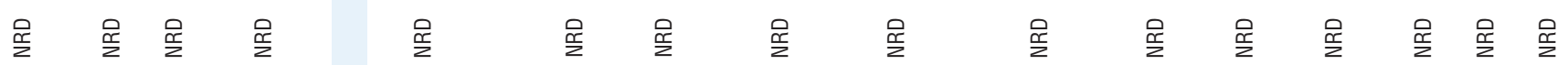

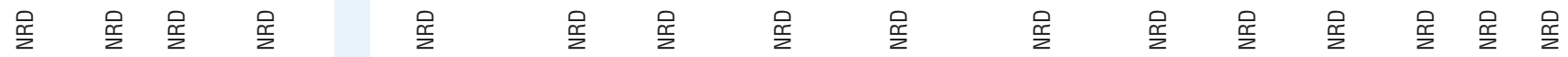

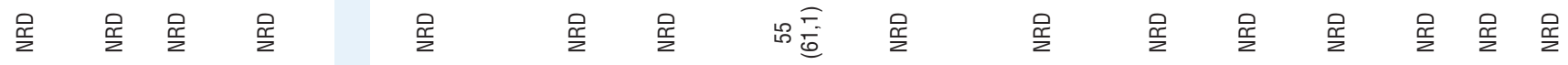

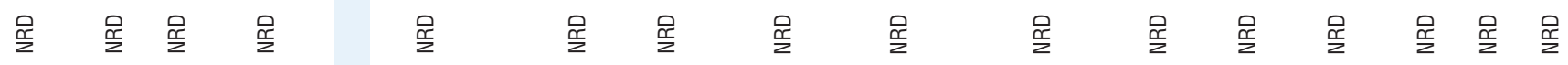

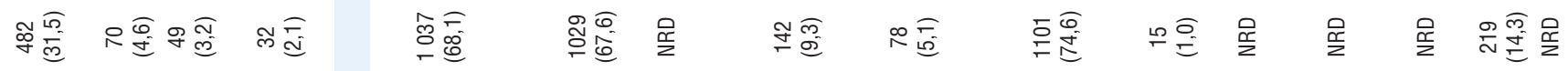

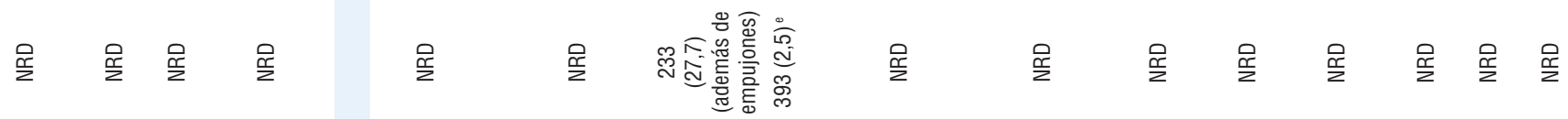

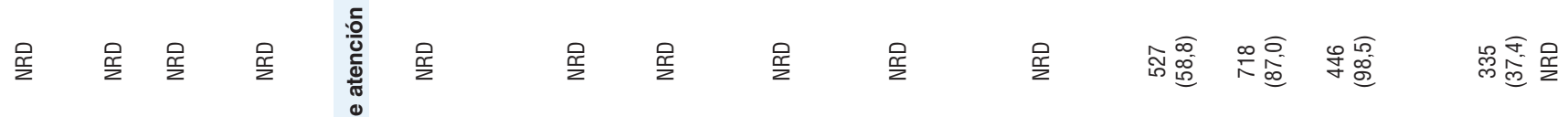

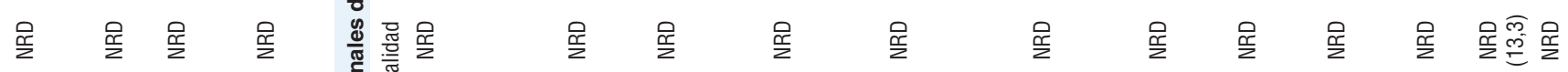

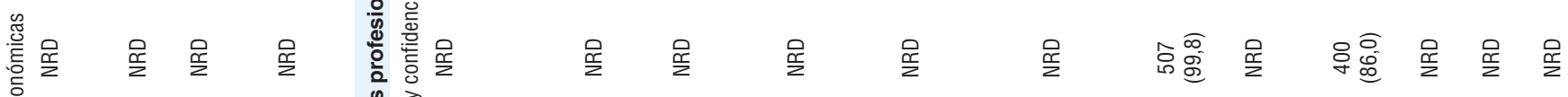

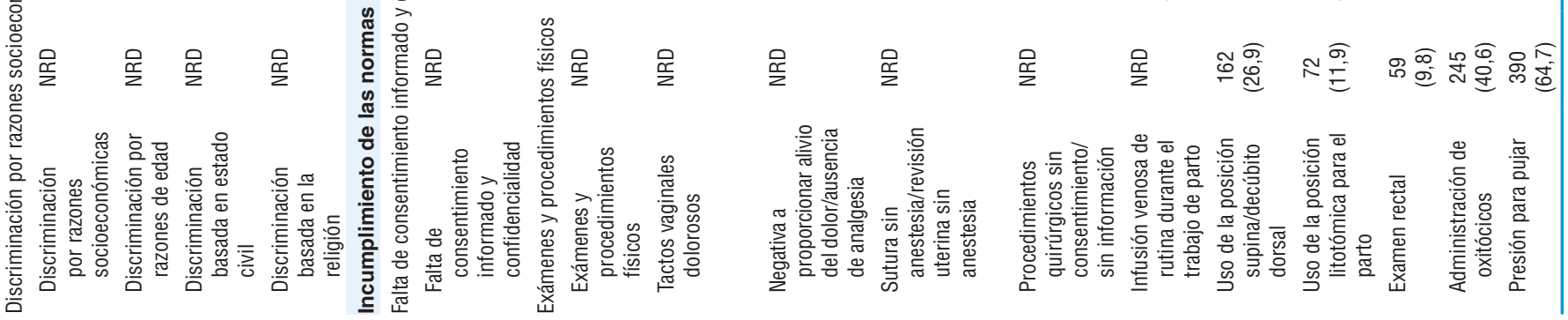




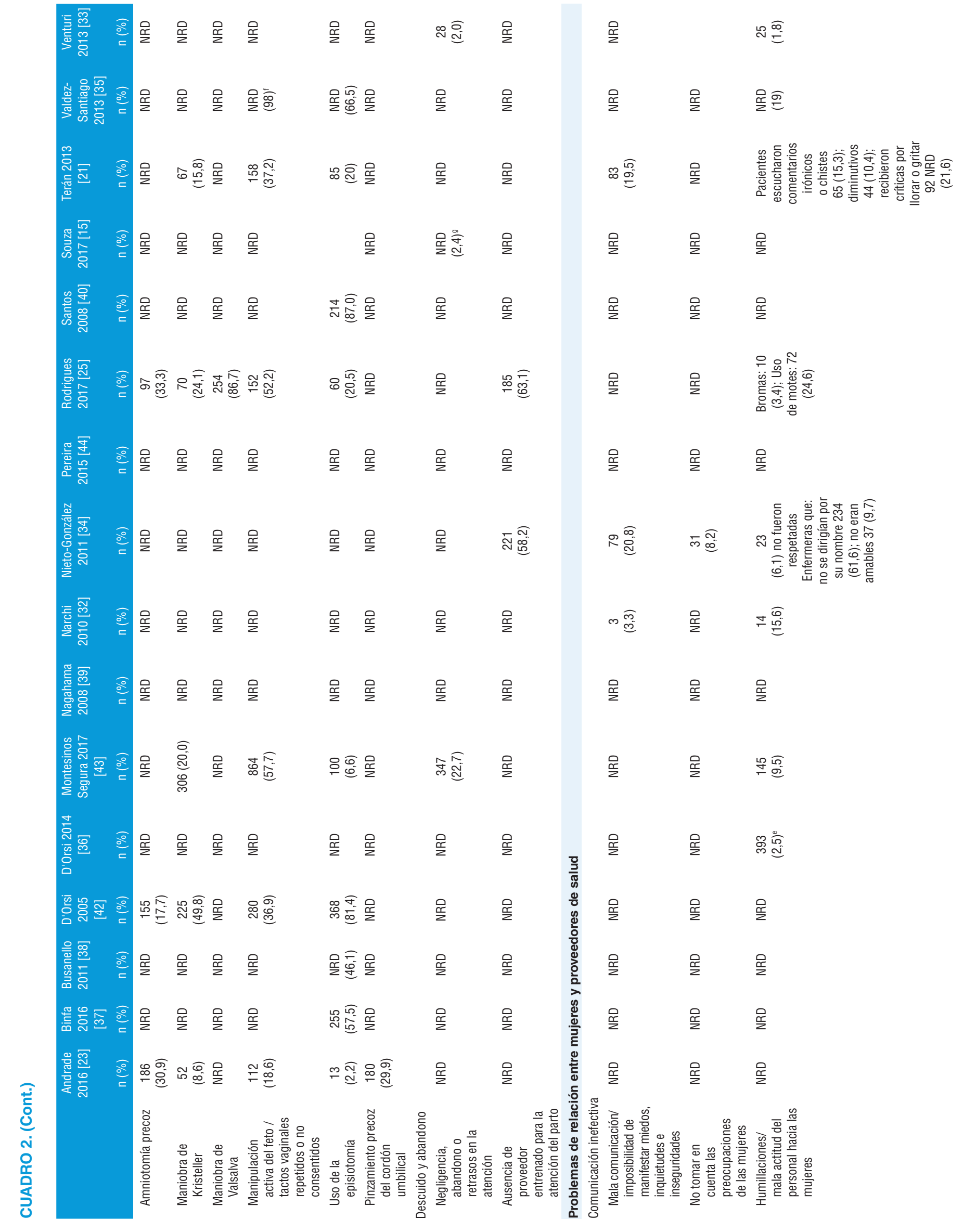




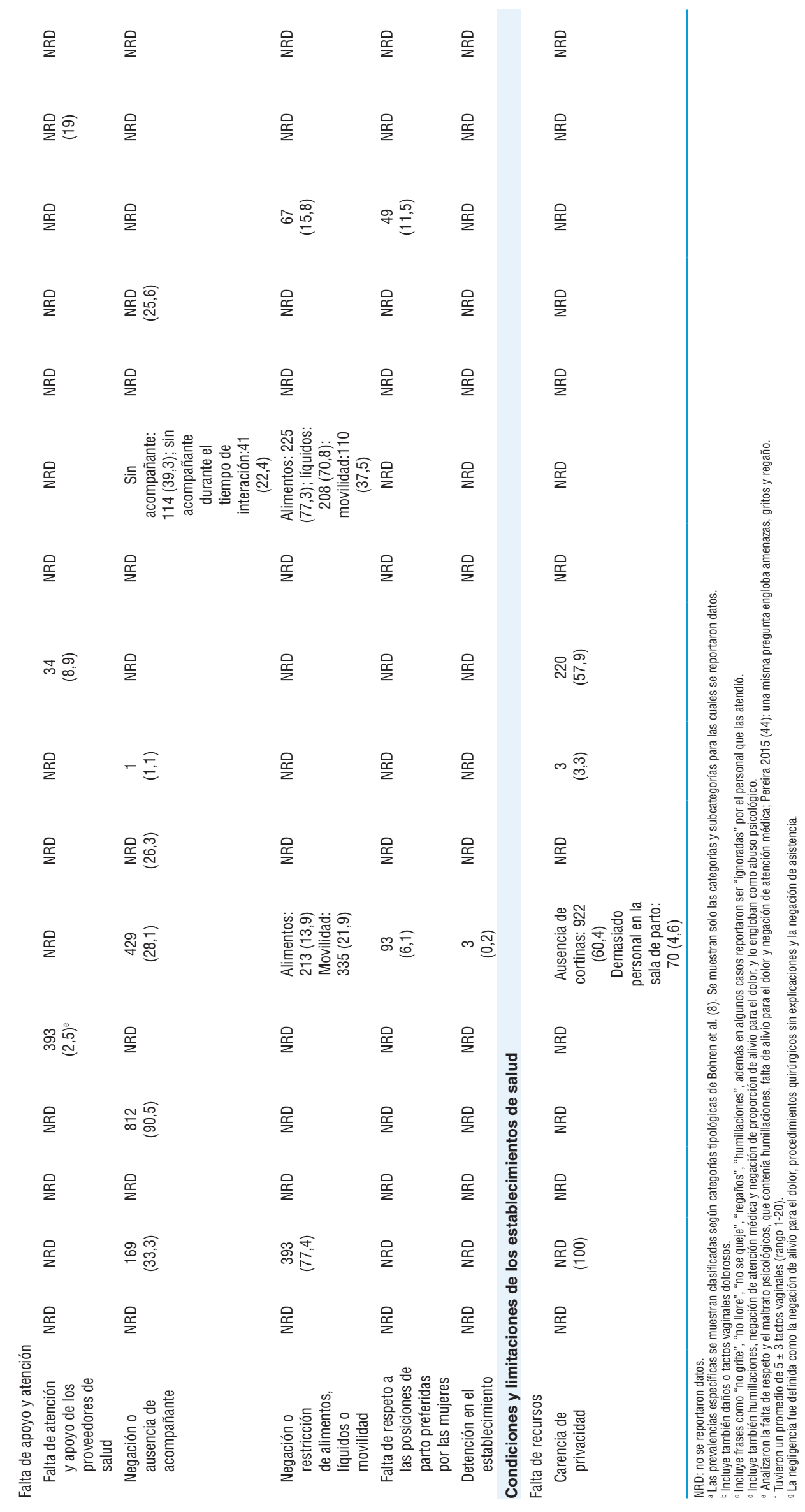


CUADRO 3. Prevalencias generales y específicas de irrespeto y maltrato en la atención institucional del aborto ${ }^{a}$

\begin{tabular}{|c|c|c|c|c|c|c|c|c|}
\hline \multirow[t]{2}{*}{ Tipo de malos tratos } & \multicolumn{2}{|c|}{$\begin{array}{l}\text { Aquino } 2012(41) \\
(n=2804)\end{array}$} & \multicolumn{2}{|c|}{$\begin{array}{l}\text { Madeiro } 2017 \\
(20)(n=78)\end{array}$} & \multicolumn{2}{|c|}{$\begin{array}{l}\text { Pereira 2015 } \\
(44)(n=101)\end{array}$} & \multicolumn{2}{|c|}{$\begin{array}{l}\text { Venturi } 2013^{c}(33) \\
(n=28 \text { reportaron } \\
\text { aborto) }\end{array}$} \\
\hline & $\mathrm{n}$ & $\%$ & $\mathrm{n}$ & $\%$ & $\mathrm{n}$ & $\%$ & $\mathrm{n}$ & $\%$ \\
\hline \multicolumn{9}{|l|}{ Abuso verbal } \\
\hline \multicolumn{9}{|l|}{ Lenguaje rudo } \\
\hline Lenguaje rudo y grosero & NRD & NRD & 18 & 23,1 & NRD & NRD & NRD & NRD \\
\hline Comentarios acusatorios o basados en prejuicios & NRD & NRD & NRD & NRD & NRD & NRD & 1 & 3,6 \\
\hline \multicolumn{9}{|l|}{ Amenazas y culpa } \\
\hline Amenaza de denuncia a la policía & NRD & NRD & 22 & 28,2 & NRD & NRD & 5 & 17,9 \\
\hline \multicolumn{9}{|l|}{ Estigma y discriminación } \\
\hline \multicolumn{9}{|l|}{ Discriminación basada en condiciones médicas } \\
\hline Juicio moral por la práctica de aborto o tratadas como sospechosas o criminales & 157 & 5,6 & 24 & 30,8 & NRD & NRD & 10 & 35,7 \\
\hline \multicolumn{9}{|l|}{ Exámenes y procedimientos físicos } \\
\hline Negativa a proporcionar alivio del dolor o ausencia de analgesia & 49 & 1,8 & 20 & 25,6 & NRD & NRD & NRD & NRD \\
\hline Procedimientos quirúrgicos sin consentimiento & NRD & NRD & 15 & 19,2 & NRD & NRD & 6 & 21,4 \\
\hline Tacto vaginal sin consentimiento & NRD & NRD & 10 & 12,8 & NRD & NRD & NRD & NRD \\
\hline Transfusión sanguínea sin consentimiento & NRD & NRD & 4 & 5,1 & NRD & NRD & NRD & NRD \\
\hline Histerectomía sin consentimiento & NRD & NRD & 1 & 1,3 & NRD & NRD & NRD & NRD \\
\hline \multicolumn{9}{|l|}{ Descuido y abandono } \\
\hline Retraso en ser atendidas & NRD & NRD & 22 & 28,2 & NRD & NRD & 5 & 17,9 \\
\hline \multicolumn{9}{|l|}{ Problemas de relación entre mujeres y proveedores de salud } \\
\hline \multicolumn{9}{|l|}{ Falta de apoyo y atención } \\
\hline Ausencia de acompañante & $\begin{array}{l}\text { Salvador } \\
\text { Recife } \\
\text { San Luis }\end{array}$ & $\begin{array}{l}49,2 \\
13,8 \\
71,2\end{array}$ & 20 & 25,6 & NRD & NRD & NRD & NRD \\
\hline \multicolumn{9}{|l|}{ Condiciones y limitaciones del sistema de salud } \\
\hline Insuficiente cambio de sábanas & $\begin{array}{l}\text { Salvador } \\
\text { Recife } \\
\text { San Luis }\end{array}$ & $\begin{array}{l}30,3 \\
46,1 \\
60,2\end{array}$ & NRD & NRD & NRD & NRD & NRD & NRD \\
\hline
\end{tabular}

a Las prevalencias específicas se muestran según categorías tipológicas de Bohren et al. (8). Se muestran solo las categorías y subcategorías para las cuales se reportaron datos.

${ }^{b}$ Los datos según las distintas categorías fueron reportados de manera agregada para partos y abortos (cuadro 2).

${ }^{c}$ En ese estudio 95 mujeres reportaron situación de aborto provocado, pero solo 28 buscaron atención médica. El denominador escogido es por tanto el de mujeres que acudieron al hospital. d 235 mujeres se sintieron discriminadas.

Las características sociodemográficas de las mujeres encuestadas se describieron en forma limitada. Solo dos estudios $(36,44)$ documentaron la prevalencia general de algún tipo de irrespeto y maltrato según características sociodemográficas. Diez estudios (56\%) reportaron la procedencia de las mujeres (entorno rural o urbano), nueve estudios (50\%) reportaron estado civil, 15 (83\%) aportaron información sobre el nivel educativo de las encuestadas, seis $(33 \%)$ documentaron la ocupación, siete $(39 \%)$ la etnia y nueve $(50 \%)$ proporcionaron datos sobre la paridad. Catorce estudios (78\%) se desarrollaron en hospitales urbanos públicos, de los cuales dos fueron universitarios. Cuatro estudios (22\%) incluyeron mujeres tanto de hospitales públicos como privados, aunque los desenlaces de interés no se reportaron por separado. Solo dos estudios (11\%) incluyeron información específica sobre el personal que brindó la atención, que señala que la mayoría fueron profesionales de medicina.

Las definiciones operativas variaron de manera sustancial entre los estudios. Los equipos de investigación usaron criterios derivados de las prácticas para el parto normal de la OMS (45), del panorama analítico propuesto por Bowser y Hill (46) y de escalas diseñadas para evaluar satisfacción con la atención.

Sin embargo, doce estudios reportaron prevalencias generales de irrespeto y maltrato medidas como la ocurrencia de alguna forma de abuso verbal, físico o ambos, trato deshumanizante o insatisfacción con la atención $(20,21,23,32-37,41,43,44)$ las cuales fueron metaanalizadas. Además, la mayoría de los estudios $(15,20,21,23,25,32-43)$ reportó prevalencias específicas, 
las cuales se agruparon por categorías tipológicas. No se realizó metaanálisis de prevalencias específicas (cuadros 2 y 3).

La medida agregada de la prevalencia general de irrespeto y maltrato en el parto y el aborto conjuntamente resultó en 39\% (IC95\%: 19-62\%, I² = 99,9\%). Esta medida no difirió de manera significativa según el tipo de atención obstétrica (parto versus aborto, $\mathrm{Q}=0,54, P=0,46$ ), el tipo de hospital (público versus ambos, público y privado, $\mathrm{Q}=1,14, P=0,29)$, el método de recopilación de datos (solo entrevista versus entrevistas y revisión del historial clínico, $\mathrm{Q}=0,33, P=0,56)$, ni la calidad de los estudios (riesgo de sesgo bajo versus moderado versus alto, $\mathrm{Q}=1,12, P=0,57)$.

La medida agregada de la prevalencia durante la atención institucional del parto (vaginal y cesárea) fue de 43\% (IC95\%: $\left.15-74 \%, \mathrm{I}^{2}=99,9 \%\right)$. Esta medida no difirió de manera significativa según el tipo de hospital $(Q=3,76, P=0,05)$, el método de recopilación de datos $(\mathrm{Q}=0,24, P=0,62)$, ni la calidad de los estudios $(\mathrm{Q}=2,34, P=0,31)$. La misma medida en los casos de aborto (espontáneo e inducido, $\mathrm{n}=4$ ) fue de 29\% (IC95\%: 10-53\%, $\left.I^{2}=96,8 \%\right)$. Esta estimación no difirió significativamente según el tipo de aborto (espontáneo vs inducido, $\mathrm{Q}=0,02, P=0,90$ ).

La heterogeneidad resultó elevada $\left(\mathrm{I}^{2}>90 \%, P<0,001\right)$. Ninguna de las variables usadas en las metarregresiones explicó este valor. Por lo tanto, se debe tener precaución a la hora de interpretar los hallazgos de los metaanálisis, ya que la alta variabilidad puede estar asociada a la disminución de la calidad de la evidencia y afectar la confiabilidad de los resultados.

Con respecto a las prevalencias específicas, se documentaron frecuencias para todas las categorías analíticas y la mayoría de las subcategorías descriptivas, si bien ninguno de los estudios por sí solo reportó datos para todas ellas. El incumplimiento de los estándares profesionales de la atención y la dificultad en la relación entre los proveedores de atención y las mujeres fueron las dos categorías más documentadas. Con relación a la primera categoría, se documentaron prevalencias entre $2,5 \%$ y $27 \%$ $(33,36)$ para tactos vaginales dolorosos y entre $19 \%(20)$ y $74,6 \%$ (43) para la falta de consentimiento informado.

En los casos de parto, la falta de consentimiento para la ligadura tubárica se documentó en 7\% y, para la histerectomía, en $4 \%$ (43). En los casos de aborto, la falta de consentimiento para la transfusión sanguínea y la histerectomía se reportaron en 5,1\% y $15,3 \%$, respectivamente (20), y para la episiotomía, entre $2 \%$ (23) y $87 \%$ (40). El uso sistemático de oxitócicos osciló entre 13\% (38) y $41 \%$ (23), la amniotomía precoz habitual entre 17,7\% (23) y 33,3\% (25) y la negativa en el uso de medicamentos para aliviar el dolor fluctuó entre $2 \%$ (41) y 61\% (32). Por su parte, la prevalencia de la maniobra de Kristeller varió entre 8,6\% (23) y 50\% (43) y la de Valsalva se documentó en $87 \%$ (25). La falta de privacidad en el parto y el aborto fue documentada entre 27\% (20) y 100\% (37).

En el ámbito de la relación de las mujeres con los proveedores, la negación de un acompañante durante el parto o el aborto fue reportada por ocho estudios con frecuencias que variaron ampliamente entre 1\% (32) y 91\% (42). Siete estudios documentaron trato humillante entre $2 \%$ (33) y $62 \%$ (34); y tres, restricciones de la movilidad entre $8 \%$ (21) y $75 \%$ (37). Otras formas de abuso en esta categoría incluyeron burlas, críticas por llorar, no ser llamadas por el nombre, uso de apodos, no tomar en cuenta las preocupaciones de la mujer, negación de alimentos o líquidos y restricción de la movilidad. Solo un estudio documentó discriminación durante el parto (43) con frecuencia de 35\% en hospitales públicos rurales, principalmente. En contraste, esta forma de abuso se reportó en todos los estudios con datos sobre aborto $(20,33,41)$ con prevalencias entre $5,6 \%$ y $35,7 \%(20,33)$. Dos de ellos con frecuencia superior a $30 \%(20,31)$.

\section{DISCUSIÓN}

Los 18 estudios incluidos en esta revisión y las medidas documentadas sugieren que el irrespeto y el maltrato son problemas prevalentes en la atención institucional del parto y el aborto en América Latina. Más de un tercio de las mujeres entrevistadas informaron haber sufrido alguna forma de irrespeto o maltrato o tener descontento con la atención recibida; y miles reportaron alguna forma de abuso físico o verbal, discriminación, incumplimiento de las normas profesionales de la atención, problemas en la relación con los proveedores de atención y derivados de las limitaciones logísticas o del funcionamiento de los establecimientos de salud.

La complejidad del fenómeno, la dificultad para establecer una definición consensuada sobre qué lo constituye, así como la falta de métodos de medición validados y comparables (47) pueden estar asociados con la escasez de los estudios sobre este tópico en la Región, la marcada variabilidad y la dificultad para la síntesis de los datos. El enfoque de algunos estudios en formas de irrespeto y maltrato con mayor prevalencia y de otros en aquellas menos prevalentes, así como la cantidad de formas del fenómeno que se incluyen en los estudios, pueden contribuir a la elevada heterogeneidad.

Además, mientras algunas formas identificadas como irrespeto y maltrato son bien reconocidas como tal (p. ej., amenazas o falta de consentimiento para procedimientos médicos), aún hay controversia sobre el reconocimiento de otras formas (p. ej., la ausencia de privacidad o la aplicación de intervenciones no basadas en evidencia científica), lo cual tiene implicaciones para la producción científica. Esto ratifica la necesidad de tener precaución a la hora de interpretar, comparar y concluir sobre los hallazgos presentados, así como la de aumentar el acervo de evidencia con metodologías estandarizadas y datos comparables que informen el desarrollo de intervenciones efectivas para el abordaje de esta problemática.

Los estudios cuantitativos sobre este tema en países de altos ingresos son escasos. No obstante, se han documentado altas variabilidades en la prevalencia también en trabajos desarrollados en África y Asia, cuyas medidas fluctúan entre 15\% y 98\% (48-50). Los estudios africanos muestran frecuencias más altas de discriminación, procedimientos sin consentimiento y, además, retención de las mujeres en instituciones hospitalarias por falta de pago.

Las exploraciones relevantes sobre los factores de riesgo y las causas de este fenómeno quedan fuera del alcance de esta revisión. Sin embargo, los modelos de formación profesional jerárquicos y débiles en competencias para la comunicación y las habilidades médico-paciente, el desempoderamiento de las mujeres, la falta de personal y de programas de educación continuada, las deficiencias logísticas, así como la ausencia de protocolos estandarizados de atención y la carencia de mecanismos de control de calidad y de rendición de cuentas en las instituciones de salud pueden estar dentro de las posibles causas del fenómeno.

Aunque los estudios de irrespeto y maltrato se han enfocado sobre todo en el parto, es importante incentivar la inclusión del aborto en estas investigaciones, ya que tres de los cuatro estudios sobre aborto describieron prácticas discriminatorias como el juicio moral, el trato de las mujeres como sospechosas o criminales, amenazas de denuncia a la policía y demora en la atención $(20,31)$. 
A este repecto, dadas las restricciones al aborto legal en ALC, es probable que varios de los abortos inducidos atendidos en las instituciones de salud hayan sido clasificados como "incompletos" y que, por lo tanto, haya error en las estimaciones de la prevalencia para abortos tanto inducidos como espontáneos.

Lo anterior cobra aún más relevancia si se tiene en cuenta que ALC no ha alcanzado las metas de salud materna proyectadas por las agendas mundiales de desarrollo a pesar de que el acceso a los servicios institucionales obstétricos y perinatales ha vertebrado las estrategias para reducir la morbimortalidad materna. Garantizar la calidad y la aceptabilidad de la atención puede acrecentar la efectividad de dicho enfoque al aparejar el aumento en el acceso con el incremento en la utilización de los servicios y disminuir así la primera demora en la atención.

Según sabemos, esta es la primera revisión sistemática en estimar la prevalencia de irrespeto y maltrato en la atención institucional del parto y del aborto en ALC. Sin embargo, hemos encontrado varios desafíos en esta tarea. A pesar de la relevancia del fenómeno, solo cinco países han recabado evidencia al respecto y todos ellos pertenecen a América Latina, con lo cual los estudios encontrados fueron escasos y los resultados circunscritos con criterio geográfico.

Además, la mayoría de los estudios no contaron con representatividad nacional, pues los hospitales, en su mayoría públicos, fueron seleccionados sobre todo por conveniencia. Por otra parte, pocos estudios reportaron el tiempo transcurrido entre el parto o aborto y el momento de la entrevista, lo que da lugar a dudas sobre la introducción de sesgo de memoria (47). Asimismo, la realización de las entrevistas en el mismo establecimiento de salud donde las mujeres pudieron haber sufrido falta de respeto y maltrato, puede aumentar el riesgo de sesgo de cortesía y subestimar la prevalencia (47). Por otra parte, la diversidad que se presenta entre y dentro de los países con respecto a las construcciones culturales que determinan las percepciones de insatisfacción y maltrato en la atención, pueden incrementar la variabilidad de los resultados (43).

Por último, la carencia de datos no permitió realizar análisis sociodemográficos. Por lo tanto, no se exploró, por ejemplo, la ocurrencia del fenómeno entre mujeres en situaciones de vulnerabilidad -indígenas, afrodescendientes, migrantes, con discapacidad y adolescentes- reconociendo que estas poblaciones están expuestas a mayor riesgo de inequidad en la atención de la salud materna y que dicha inequidad se relaciona no solo con menor disponibilidad y acceso a los servicios, sino también con una calidad sistemáticamente desigual de estos.

\section{CONCLUSIONES}

Los datos extraídos en esta revisión indican que el irrespeto y el maltrato durante la atención institucional del parto y del aborto son prevalentes en la Región. Sin embargo, dada la elevada heterogeneidad documentada en los estudios incluidos, no fue posible obtener una estimación de alta calidad sobre la magnitud regional del fenómeno. Los datos reunidos y analizados en este trabajo pueden incentivar la producción de investigaciones regionales con enfoque de salud pública que produzcan medidas representativas, rigurosas y comparables sobre este fenómeno. Esto es en especial relevante para la implementación de servicios de salud perinatal en general, y del parto y el aborto en particular, que sean aceptables y centrados en las mujeres.

Alcanzar esta meta requiere incorporar estrategias de calidad que apunten no solo a la implementación de servicios oportunos y seguros y a la mejora en las condiciones de los establecimientos y los sistemas de salud, sino que, en consonancia con las recomendaciones basadas en la evidencia, promuevan la participación de las mujeres en el diseño de los servicios, respondan a sus aspiraciones, necesidades y percepciones, cuenten con enfoques diferenciales y garanticen sus derechos a una atención respetuosa y libre de discriminación. Lo anterior es imprescindible para reducir la morbimortalidad materna e infantil, posibilitar que las mujeres, los niños y las niñas prosperen y alcancen su máximo potencial y contribuir, por ende, al desarrollo regional.

Contribución de los autores. AG y JPS concibieron el estudio; $\mathrm{MP}, \mathrm{CH}$, SM y VP seleccionaron estudios; MP extrajo los datos; $\mathrm{CH}$ y MP escribieron el manuscrito original; AG, LR, RVS, LAM, ARC, MPP, and RGPL revisaron el manuscrito. Todos los autores revisaron y aprobaron la versión final.

Agradecimientos. A Meghan Bohren, quien colaboró en el proceso de elaboración de las estrategias de búsqueda, y a Eleonora D’Orsi y la Fundación Perseu Abramo (en la persona de Matheus Toledo) por su valiosa colaboración.

\section{Conflicto de intereses. Ninguno declarado por los autores.}

Declaración. Las opiniones expresadas en este manuscrito son responsabilidad del autor y no reflejan necesariamente los criterios ni la política de la RPSP/PAJPH y / o de la OPS.

\section{REFERENCIAS}

1. United Nations High Commissioner for Human Right: Practices in adopting a human rights-based approach to eliminate preventable maternal mortality. Report of the Office of the United Nations High Commissioner for Human Rights. New York: UN; 2011.

2. Organización de las Naciones Unidas: Estrategia Mundial para la Salud de la Mujer, el Niño y el Adolescente (2016-2030). Sobrevivir, prosperar, transformar. Nueva York: ONU; 2016

3. Organización de las Naciones Unidas: La Agenda 2030 y los Objetivos de Desarrollo Sostenible Una oportunidad para América Latina y el Caribe. Santiago de Chile: CEPAL; 2016

4. Silver K and Singer P: SDGS: Start with maternal, newborn, and child health cluster. Lancet. 2014;384(9948):1093-4

5. Freedman LP, Ramsey K, Abuya T, Bellows B, Ndwiga C, Warren CE, Kujawski S, Moyo W, Kruk ME, Mbaruku G: Defining disrespect and abuse of women in childbirth: a research, policy and rights agenda. Bull World Health Organ. 2014;92(12):915-7.

6. Organización Mundial de la Salud: Prevención y erradicación de la falta de respeto y el maltrato durante la atención del parto en centros de salud, 2014. Consultado 15 marzo 2017. Disponible en: <http:// apps.who.int/iris/bitstream/10665/134590/1/WHO_RHR_14.23 spa.pdf?ua $=1 \& u a=1$

7. Cariny Ciello C, Carvalho C, Kondo C, Delage D, Niy D, Werner L, Santos K: Parirás com dor: Dossiê elaborado pela Rede Parto do Princípio para a CPMI da Violência contra as mulheres. Brasilia: Mulheres em Rede pela Maternidade Ativa; 2012.

8. Bohren MA, Vogel JP, Hunter EC, Lutsiv O, Makh SK, Souza JP, Aguiar C, Saraiva Coneglian F, Diniz AL, Tuncalp O et al: The Mistreatment of Women during Childbirth in Health 
Facilities Globally: A Mixed-Methods Systematic Review. PLoS Med. 2015;12(6):e1001847; discussion e1001847.

9. Small R, Yelland J, Lumley J, Brown S, Liamputtong P: Immigrant women's views about care during labor and birth: an Australian study of Vietnamese, Turkish, and Filipino women. Birth (Berkeley, Calif). 2002;29(4):266-77.

10. d'Oliveira AF, Diniz SG, Schraiber LB: Violence against women in health-care institutions: an emerging problem. Lancet (London, England) 2002; 359(9318):1681-85.

11. Castro Pérez R, López Gómez A: Poder médico y ciudadanía: el conflicto social de los profesionales de la salud con los derechos reproductivos en América Latina. Avances y desafíos en la investigación Montevideo: Universidad de la República; México: UNAM, CRIM; 2010.

12. Bohren MA, Hunter EC, Munthe-Kaas HM, Souza JP, Vogel JP, Gulmezoglu AM: Facilitators and barriers to facility-based delivery in low- and middle-income countries: a qualitative evidence synthesis. Reprod Health. 2014;11(1):71.

13. Thaddeus S, Maine D: Too far to walk: maternal mortality in context. Soc Sci Med (1982). 1994;38(8):1091-1110.

14. Pacagnella RC, Cecatti JG, Parpinelli MA, Sousa MH, Haddad SM, Costa ML, Souza JP, Pattinson RC: Delays in receiving obstetric care and poor maternal outcomes: results from a national multicentre cross-sectional study. BMC Pregnancy Childbirth 2014:14:159.

15. Souza KJd, Rattner D, Gubert MB: Institutional violence and quality of service in obstetrics are associated with postpartum depression. Rev Saude Publica. 2017; 51.

16. Silva A, Góis J: Entre a acusação e a aceitação: um estudo das percepções de uma equipe de saúde sobre a mulher gestante com HIV / Aids. Niterói. 2011;11(2): 9-28.

17. Freedman LP, Ramsey K, Abuya T, Bellows B, Ndwiga C, Warren CE, Kujawski S, Moyo W, Kruk ME, Mbaruku G: Defining disrespect and abuse of women in childbirth: a research, policy and rights agenda. Bull World Health Organ. 2014; 92(12):915-7.

18. Freedman LP, Kruk ME: Disrespect and abuse of women in childbirth: challenging the global quality and accountability agendas. Lancet (London, England) 2014; 384(9948):e42-4.

19. Windau-Melmer T: A Guide for Advocating for Respectful Maternity Care; Washington DC: Futures Group, Health Policy Project; 2013.

20. Madeiro AP, Rufino AC: Maus-tratos e discriminação na assistência ao aborto provocado: a percepção das mulheres em Teresina, Piauí, Brasil. Cien Saude Colet. 2017;22(8):2771-80.

21. Terán P, Castellanos C, Blanco M, Ramos D: Violencia obstétrica: percepción de las usuárias. Revista de Obstetricia y Ginecologia de Venezuela. 2013;73(3):171-80.

22. Brasil MdS (MS): Violência intrafamiliar: orientações para prática em serviço. Brasilia: Ministério da Saúde (MS); 2001.

23. Andrade PdON, Silva JQPd, Diniz CMM, Caminha MdFC: Fatores associados à violência obstétrica na assistência ao parto vaginal em uma maternidade de alta complexidade em Recife, Pernambuco. Rev Bras Saude Mater Infant. 2016; 16(1):29-37.

24. Chadwick RJ: Obstetric violence in South Africa. S Afr Med J. 2016;106(5):5-8.

25. Rodrigues F, Gama S, Magalhaes P, Freitas A, Da Silva V e Almeida P.: Violência obstétrica no processo de parturição em maternidades viculadas à Rede Cegonha. Reprod Climat. 2017;32(2):78-84

26. Sadler M, Santos MJ, Ruiz-Berdun D, Rojas GL, Skoko E, Gillen P, Clausen JA: Moving beyond disrespect and abuse: addressing the structural dimensions of obstetric violence. Reprod Health Matters. 2016;24(47):47-55.

27. Madeira S, Pileggi V, Souza JP: Abuse and disrespect in childbirth process and abortion situation in Latin America and the Caribbean-systematic review protocol. Syst Rev. 2017;6(1):152.

28. Hoy D, Brooks P, Woolf A, Blyth F, March L, Bain C, Baker P, Smith E, Buchbinder R: Assessing risk of bias in prevalence studies: modification of an existing tool and evidence of interrater agreement. J Clin Epidemiol. 2012;65(9):934-9.

29. Barendregt JJ, Doi SA, Lee YY, Norman RE, Vos T: Meta-analysis of prevalence. J Epidemiol Community Health. 2013;67(11):974-8.

30. Miller JJ: The Inverse of the Freeman - Tukey Double Arcsine Transformation. The Am Stat. 1978;32(4):138.

31. Moher D, Liberati A, Tetzlaff J, Altman DG: Preferred reporting items for systematic reviews and meta-analyses: the PRISMA statement. Int J Surg. 2010;8(5):336-41.
32. Narchi NZ, Diniz CSG, Azenha CdAV, Scheneck CA: Women's satisfaction with childbirth' experience in different models of care: a descriptive study. Online Braz J Nurs. 2010;9(2).

33. Venturi G y Godinho T: Mulheres brasileiras e gênero nos espaços público e privado: uma década de mudanças na opinião pública. São Paulo: Fundação Perseu Abramo; 2013.

34. Nieto-González LA R-QM, Córdoba-Ávila MA, Campos-Castolo M: Percepción del trato digno por la mujer embarazada en la atención obstétrica de enfermería. Rev CONAMED México. 2011;16(Supl.1):S5-S11.

35. Valdez-Santiago R, Hidalgo-Solórzano E, Mojarro-Iñiguez M, Arenas-Monreal LM: Nueva evidencia a un viejo problema: el abuso de las mujeres en las salas de parto. CONAMED. 2013, 18(1):14-20.

36. D'Orsi E BO, Diniz CSG, Aguiar JM, Gusman CR, Torres JA, Angulo-Tuesta A, Rattner D, Domingues RMSM: Desigualdades sociais e satisfação das mulheres com o atendimento ao parto no Brasil: estudo nacional de base hospitalar - Inquérito Nacional Nascer no Brasil. Cad Saude Publica. 2014; 30(Suplemento):S154-68.

37. Binfa L, Pantoja L, Ortiz J, Gurovich M, Cavada G, Foster J: Assessment of the implementation of the model of integrated and humanised midwifery health services in Chile. Midwifery. 2016;35:53-61.

38. Busanello J, Kerber NP, Mendoza-Sassi RA, Mano Pde S, Susin LR, Goncalves BG: [Humanized attention to parturition of adolescents: analysis of practices developed in an obstetric center]. Rev Bras Enferm. 2011;64(5):824-32.

39. Nagahama EE, Santiago SM: [Childbirth practices and challenges for humanization of care in two public hospitals in Southern Brazil]. Cad Saude Publica. 2008; 24(8):1859-68.

40. Santos JdO, Bolanho IC, Mota JQCd, Oliveira MAd, Coleoni L: Frequency of perineal lesions occurred during natural child labour in a hospital institution. Esc Anna Nery Rev Enferm. 2008;12(4):658-63.

41. Aquino EM, Menezes G, Barreto-de-Araujo TV, Alves MT, Alves SV, de Almeida Mda C, Schiavo E, Lima LP, de Menezes CA, Marinho LF et al.: [Quality of abortion care in the Unified Health System of Northeastern Brazil: what do women say?]. Cien Saude Colet. 2012;17(7):1765-76.

42. d'Orsi E, Chor D, Giffin K, Angulo-Tuesta A, Barbosa GP, Gama Ade S, Reis AC, Hartz Z: [Quality of birth care in maternity hospitals of Rio de Janeiro, Brazil]. Rev Saude Publica. 2005;39(4):645-54.

43. Montesinos-Segura R, Urrunaga-Pastor D, Mendoza-Chuctaya G, Taype-Rondan A, Helguero-Santin LM, Martinez-Ninanqui FW, Centeno DL, Jimenez-Meza Y, Taminche-Canayo RC, Paucar-Tito L et al: Disrespect and abuse during childbirth in fourteen hospitals in nine cities of Peru. Int J Gynaecol Obstet. 2018; Feb;140(2):184-190. doi: 10.1002/ijgo.12353. Epub 2017 Nov 9; 44. Pereira CJ, Domínguez AL, Toro Merlo J: Violencia obstétrica desde la perspectiva de la paciente. Rev Obstet Ginecol Venez. 2015;75(2):81-90.

45. Organización Mundial de la Salud. Recomendaciones para la conducción del trabajo de parto normal. Ginebra: OMS, 2015.

46. Bowser D HK: Exploring evidence for disrespect and abuse in facility-based childbirth: Report of a landscape analysis. Washington DC: USAID; 2010.

47. Savage V, Castro A: Measuring mistreatment of women during childbirth: a review of terminology and methodological approaches. Reprod Health. 2017;14(1):138.

48. Okafor, II, Ugwu EO, Obi SN: Disrespect and abuse during facility-based childbirth in a low-income country. J Int Fed Gynaecol Obstet. 2015;128(2):110-3.

49. Abuya T, Warren CE, Miller N, Njuki R, Ndwiga C, Maranga A, Mbehero F, Njeru A, Bellows B: Exploring the prevalence of disrespect and abuse during childbirth in Kenya. PloS One. 2015;10 (4): $\mathrm{e} 0123606$.

50. Raj A, Dey A, Boyce S, Seth A, Bora S, Chandurkar D, Hay K, Singh K, Das AK, Chakraverty A et. al.: Associations Between Mistreatment by a Provider during Childbirth and Maternal Health Complications in Uttar Pradesh, India. Matern Child Health J. 2017 Sep;21(9):1821-1833. doi: 10.1007/s10995-017-2298-8 2017

Manuscrito recibido el 11 de setiembre de 2018. Aceptado para su publicación, tras revisión, el 25 de enero de 2019. 


\section{Disrespect and abuse during childbirth and abortion in Latin America: systematic review and meta-analysis}

ABSTRACT Objective. This review synthesizes the evidence (quantitative, general, and by typological categories) of disrespect and abuse during childbirth and abortion in health facilities in Latin America and the Caribbean.

Methods. Systematic searches identified 18 primary studies. $Q$ and $\mathrm{I}^{2}$ were calculated, meta-analyses and meta-regressions were performed, and subgroups were analyzed using a DerSimonian and Laird random-effects model grouped by inverse variance and the Freeman-Tukey double arcsine transformation.

Results. Studies conducted in five Latin American countries were identified. No studies from the Caribbean were found. The aggregate prevalence of disrespect and abuse during childbirth and abortion was $39 \%$. The aggregated prevalence of the phenomenon in childbirth was $43 \%$ and $29 \%$ during abortion. The high heterogeneity made it impossible to generate aggregate measures according to typological categories. Nevertheless, the frequencies of specific forms of the phenomenon were grouped typologically.

Conclusions. The evidence suggests that disrespect and abuse during childbirth and abortion care are human-rights and public-health problems that are prevalent in some countries of the Region. It is necessary to reach international consensus on the definition and operationalization of this problem and to develop standardized methods for its measurement. Doing so is essential in order to achieve the targets of the 2030 Agenda related to reducing maternal and newborn morbidity and mortality and eliminating all forms of violence and discrimination against women.

Keywords Violence against women; humanizing delivery; women's health services; abortion; parturition.

\section{Desrespeito e maus-tratos durante o parto e o aborto na América Latina: revisão sistemática e meta-análise}

RESUMO Objetivo. Esta revisão sintetiza as evidências quantitativas, gerais e desagregadas por categorias tipológicas do desrespeito e maus-tratos na atenção institucional ao parto e ao aborto na América Latina e Caribe.

Métodos. Dezoito estudos primários foram identificados por meio de buscas sistemáticas. Foi feito o cálculo de $Q$ e $I^{2}$ e realizadas meta-análises, metarregressões e análises de subgrupos com um modelo de DerSimonian e Laird de efeitos aleatórios agrupados com variância inversa e transformação de Freeman-Tukey (duplo arco-seno).

Resultados. Foram identificados estudos realizados em cinco países da América Latina. Não foi identificado nenhum estudo no Caribe. Observou-se uma prevalência agregada de 39\% de desrespeito e maus-tratos durante o parto e o aborto. A medida agregada para este fenômeno durante o parto foi $43 \%$ e a medida agregada nos casos de aborto foi $29 \%$. Devido à alta heterogeneidade, não foi possível gerar medidas agregadas segundo categorias tipológicas. No entanto, são descritas as frequências de formas específicas do fenômeno agrupadas tipologicamente.

Conclusões. As evidências indicam que o desrespeito e os maus-tratos na atenção ao parto e ao aborto são uma questão de direitos humanos e de saúde pública prevalente em alguns países da Região. É preciso chegar a um consenso internacional sobre a definição e a operacionalização deste problema e elaborar métodos padronizados para mensurá-lo. Isso é imprescindível para o alcance das metas da Agenda 2030 relativas à redução da morbidade e mortalidade materna e perinatal e à eliminação de todas as formas de violência e discriminação contra a mulher.

Palavras-chave Violência contra a mulher; parto humanizado; serviços de saúde da mulher; aborto; parto. 